\title{
Adoption des nouvelles technologies rizicoles sur l'efficacité technique au Sénégal
}

\section{Adoption of new rice technologies on technical efficiency in Senegal}

\author{
Babacar Ndiaye ${ }^{1}$, Albertine Bayompe Kabou ${ }^{2}$ \\ ${ }^{1}$ Département Economie Gestion, Université Amadou Mahtar Mbow, Sénégal, babacar.ndiaye@uam.edu.sn \\ ${ }^{2}$ Département Economie Gestion, Université Cheikh Anta Diop, Sénégal, A.KABOU679@zig.univ.sn
}

RÉSUMÉ. Ce papier analyse l'impact de l'adoption des nouvelles technologies rizicoles sur l'efficacité technique des agriculteurs au Sénégal. En désagrégeant les différentes possibilités ou traitement en trois niveaux à l'aide d'un traitement multiple, les résultats montrent que, d'une part, le premier niveau de traitement T1 (engrais) n'a pas d'impact sur l'efficacité technique des riziculteurs et, d'autre part, le deuxième niveau de traitement T2 (engrais et semence améliorée) et le troisième niveau de traitement T3 (engrais, semence améliorée et équipement motorisé) impactent sur l'efficacité technique des riziculteurs respectivement de $9,7 \%$ et de $12,1 \%$.

ABSTRACT. This paper analyzes the impact of the adoption of new rice technologies on the technical efficiency of farmers in Senegal. By disaggregating the different possibilities or treatment into three levels using multiple treatment, the results show that, on the one hand, the first treatment level T1 (fertilizer) has no impact on the technical efficiency of the rice farmers and, on the other hand, the second treatment level T2 (fertilizer and improved seed) and the third treatment level T3 (fertilizer, improved seed and motorized equipment) impact on the technical efficiency of rice farmers by $9.7 \%$ and $12.1 \%$, respectively.

MOTS-CLÉS. Innovation technologique, efficacité technique, agriculture, riz, Sénégal.

KEYWORDS. Technological innovation, technical efficiency, agriculture, rice, Senegal.

JEL CLASSIFICATION : D61, Q16, Q55

\section{Introduction}

Dans la majorité des pays d'Asie, d'Afrique, d'Amérique et des Caraïbes, le riz constitue la nourriture de base [OEC 17]. Le riz est la céréale la plus consommée au monde et occupe le troisième rang des céréales produites au niveau mondial après le blé et le maïs [SEC 12]. L'Afrique est l'un des premiers importateurs de riz au monde, $40 \%$ de ses besoins sont assurés par les importations provenant d'Asie [BOU 13]. Selon les prévisions de la Banque mondiale, la production de riz usinée est estimée à 509,2 millions de tonnes [FAO 20].

En Afrique de l'ouest, le Sénégal est l'un des pays où la consommation de riz est très élevée avec une moyenne annuelle par habitant estimée à $90 \mathrm{~kg}$. En effet, depuis la colonisation, la consommation des brisures de riz est introduite dans les habitudes de consommation des sénégalais jusqu'à devenir un aliment de base. La consommation annuelle est estimée à 1.080 .000 tonnes, dont 650.000 tonnes issues de l'importation et 430.000 tonnes issues de la production locale [PRA 14].

Pourtant, le gouvernement du Sénégal a consenti beaucoup d'investissement dans le secteur durant ces dernières années avec successivement le Programme National d'Autosuffisance en Riz (PNAR) en 2008, la Grande Offensive Agricole pour la Nourriture et l'Abondance (GOANA) en 2010 et le Programme de Relance et d'Accélération de la Cadence de l'agriculture Sénégalais (PRACAS) en 2014. Toutefois, ces programmes n'ont pas atteint les objectifs en termes d'autosuffisance, de sécurité et de souveraineté alimentaires [NGO 16]. Par conséquent, les opportunités offertes par les innovations technologiques vis-à-vis des semences améliorées, de l'engrais et des matériels agricoles deviennent des opportunités essentielles pour améliorer l'efficacité technique dans les économies en développement [NKA 04]. Le concept d'efficacité technique se définit comme une situation dans laquelle une aussi grande quantité d'output est produite à partir d'un ensemble d'inputs donné [FAR 
57]. Depuis lors, ce concept a évolué selon des applications relatives à plusieurs domaines [MAR 97] ; [DEM 05] ; [NUA 10].

Certains auteurs [KHA 17, CHU 20, GER 20] expliquent la faiblesse de la productivité dans la plupart des pays en développement par le manque d'efficacité technique. Cette situation s'explique par le fait que la plupart des agriculteurs dans les pays en développement opèrent en deçà de leur capacité de production potentielle [KEA 09, THI 95]. C'est le cas au Sénégal où, en moyenne, les riziculteurs du bassin du Fleuve Sénégal ne sont pas efficaces [NGO 16]. Ces auteurs ont montré que les riziculteurs enregistrent un écart de 30\% entre la production observée et celle réalisée. Cet écart s'explique par le fait que la majorité des producteurs ne se situent pas sur la frontière de production et de coûts. Ainsi, l'accroissement de la productivité agricole passe par l'introduction de changement technologique [NUA 06, GIL 90]. En utilisant des données de pannel sur huit pays d'Afrique francophone sur la période 1970-2000, le changement technologique a été la principale cause de l'échec de la productivité totale des facteurs de ces pays [NKA 04].

De surcroit, l'adoption des nouvelles technologies agricoles est considérée comme une alternative de sortie de la pauvreté pour plusieurs économies en développement [BAN 06]. Les études empiriques sur l'évaluation d'impact montrent que l'adoption de ces technologies est conditionnée par les variables observables et les variables inobservables [ADE 93, ADE 95, ALE 06, HAI 14]. Ainsi, plusieurs travaux ont ténté d'évaluer l'impact de l'adoption des nouvelles technologies en utilisant diverses méthodes d'éstimation comme celle relative à la regression binomiale [HEC 01]. D'ailleurs, la prise en compte de l'hétérogénéité dans l'adoption des nouvelles technologies est connue comme une caractéristique importante des méthodes d'évaluation d'impact des programmes [HEC 98, DEB 06]. Néanmoins, des travaux plus récents sur les progrès technologiques sont proposés avec des modèles d'apprentissage automatique pour identifier les profils d'adoption de plusieurs technologies agricoles par les exploitants [LAM 19].

Notre article se démarque des travaux [DUF 11, DUV 17] qui ont défini le traitement, c'est-à-dire la participation au projet ou programme de façon binaire (par oui ou non). En considérant, les niveaux de traitement comme l'adoption des nouvelles technologies rizicoles, nous avons désagrégé les différentes possibilités ou traitement qu'offrent les programmes en utilisant la régression logistique multinomiale. Ces traitements sont l'engrais, les semences améliorées et les équipements motorisés. Ainsi le niveau de traitement $\mathrm{T} 1$ concerne les riziculteurs qui ont seulement adopté l'engrais, le niveau de traitement T2 est l'ensemble des riziculteurs qui ont adopté simultanément l'engrais et les semences améliorées et le dernier niveau de traitement T3 regroupe les riziculteurs qui ont combiné l'engrais, les semences améliorées et l'équipement motorisé.

Cet article est organisé comme suit. La première section présente le cadre théorique des méthodes d'estimation. La deuxième section présente les spécifications du modèle pour détecter l'impact de chaque niveau de traitement sur l'efficacité technique des riziculteurs. La conclusion présente les discussions et les perspectives de recherche.

\section{Cadre théorique}

\subsection{Approche théorique des déterminants de l'adoption}

La littérature sur les déterminants de l'adoption met en évidence plusieurs caractéristiques qui influencent le statut d'adoption. L'adoption fait référence à plusieurs concepts dans la littérature [HOC 18]. Amed et al. [AME 17] soutiennent que l'adoption est une decicion prise le plus souvent par la direction d'une entreprise afin d'indiquer aux travailleurs l'introduction d'une nouvelle technologie dans leur travail. Elle est considèrée comme un processus qui consiste à intégrer une innovation [ROG 03] qui est une combinaison entre les concepts d'implémentation et de diffusion [SUC 15]. Ainsi, l'adoption des nouvelles technologies constitue une forme d' «innovation au niveau des procédés» 
(méthodes de gestion et de production) par opposition à «innovation au niveau des produits» (création de nouveaux produits dont certaines caractéristiques sont différentes des produits précédents) [LEF 90].

Le rôle de la femme a été démontré dans le processus d'adoption [ADE 95]. Ces auteurs montrent qu'en Afrique de l'Ouest, les femmes sont les mieux placées pour apprécier la qualité des produits dérivés des nouvelles technologies. Au Malawi, Chirwa [CHI 05] trouve que les ménages dirigés par des femmes sont les moins impactés par rapport l'adoption à des nouvelles technologies. En effet, les ménages dirigés par des femmes sont plus susceptibles d'être pauvres et d'avoir des ressources limitées, ce qui réduit leur capacité à adopter les nouvelles technologies.

Concernant l'âge, Alene et Manyong [ALE 05] montrent que les agriculteurs relativement âgés sont moins outillés à utiliser certaines nouvelles technologies de façon efficience. En revanche, l'émergence de nouveaux outils a permis à cette catégorie de personnes d'adopter plus facilement les nouvelles technologies [ADE 95].

Plusieurs facteurs financiers peuvent également motiver l'adoption des nouvelles technologies. Des travaux ont montré que dans les pays en développement, l'inexistence ou l'imperfection du marché de l'assurance constitue un véritable frein à l'adoption des nouvelles technologies [UDR 10]. Il s'y ajoute les difficultés d'accès au crédit qui peuvent aussi constituées un handicap dans l'adoption des nouvelles technologies surtout pendant les périodes hors récoltes [DUF 11]. De ce fait, les agriculteurs sont souvent confrontés à des problèmes de liquidité. Pour rémédier à ces contraintes, la disponibilité et l'accès au crédit permettront de renforcer l'utilisation de certains intrants [ZEL 98, ALE 06, HAI 14]).

Durant la révolution verte en Chine (1960-1990), coincidant à une volonté politique et industrielle, appuyée sur les progrès scientifiques et techniques réalisés dans le domaine agricole, l'adoption des nouvelles technologies agricoles a conduit à l'augmentation significative de la productivité agricole [BAN 08]. Dans le même sens, de nombreux travaux montrent que l'adoption des technologies améliorées permet d'accroître la productivité agricole [MEN 07, SEC 12].

\subsection{Approche empirique}

A travers la littérature économique, plusieurs travaux ont abordé le choix d'adoption de nouvelles technologies par les agriculteurs. Adékambi [ADE 05], en mettant particulièrement l'accent sur l'investissement dans la scolarité et la santé des enfants, évalue l'impact socio-économique des variétés améliorées de riz au Bénin en utilisant la comparaison simple entre les adoptants et les non-adoptants. Mayen et al. [MAY 10] ont utilisé le score de propension qui correspond à l'analyse des frontières de production pour comparer la productivité entre les fermes conventionnelles et laitières aux Etats-Unis. Dans la même approche, Martey et al. [MAR 15] évaluent l'impact de la participation d'un projet de mentorat sur l'efficacité agricole. Villano et al. [VIL 95] utilisent l'appariement des scores de propension dans l'analyse des frontières de production pour comparer l'inefficacité technique entre les adoptants et les non-adoptants de semences certifiées aux Philippines.

L'efficacité technique permet de soutenir que le producteur a une production potentielle maximale, compte tenu des facteurs de production (intrants) dont il dispose. Les méthodes de calcul de l'efficacité technique peuvent être regroupées en deux grandes familles : les méthodes paramétriques et celles non paramétriques [NGO 16]. Cependant, dans le secteur agricole, l'estimation par les frontières de production paramétrique est plus appropriée [NGO 16, AIG 77, MEE 77]. Contrairement à la méthode par enveloppement des processus agricoles, celle-ci inclut les déterminants de l'inefficacité technique des ménages agricoles [LAM 19].

Afin d'évaluer l'impact de l'adoption des nouvelles technologies sur l'efficacité, il est important de se référer à la littérature sur l'adoption qui fait référence à la décision de mettre en œuvre des propositions techniques nouvelles qui dépendent des caractéristiques socio-économiques de potentiels adoptants [FED 93, ROG 03]. Au-delà de cette approche, les propositions techniques dépendent également de la compatibilité des caractéristiques des innovations à l'environnement institutionnel 
(normes, règles, valeurs), technologique (systèmes techniques existant, savoir-faire, risque) et économique (accessibilité des facteurs de production nécessaires) des potentiels adoptants, mais aussi de la perception qu'ils ont des caractéristiques des innovations qui leurs sont proposées [ROG 03]. Ainsi, l'attribution du traitement est distribuée de manière non aléatoire dans la population et que le motif de l'adoption est déterminé, soit par des variables observables, soit par des variables inobservables. Le défi est d'identifier un contrefactuel, c'est-à-dire un groupe de comparaison approprié et semblable au groupe traité.

Même si l'approche de l'appariement des scores de propension est la méthode la plus répandue en évaluation des politiques publiques, cette dernière conduit à des estimations biaisées inefficaces et inexactes [LAM 16]. En effet, la méthode d'appariement des scores de propension est une probabilité prédite d'attribution de traitement en fonction des caractéristiques observées. Donc, la méthode d'appariement par le score de propension permet d'éliminer le biais induit par les caractéristiques observables [MUL 12]. Ces auteurs utilisent cette méthode pour évaluer l'impact potentiel de l'adoption de technologies agricoles sur l'état de la consommation alimentaire des ménages.

Cependant, plusieurs limites sont soulignées sur les régressions simples car le biais lié à l'autosélection des données d'observations collectées n'est pas éliminé [RUB 74, RUB 83, HEC 05]. Ainsi, pour la prise en compte de ce biais, Mulugeta et Hundie [MUL 12] ont développé des théories à travers les méthodes expérimentales et quasi-expérimentales pour régler le problème d'auto-sélection. La limite de cette méthode est qu'elle ne contrôle que les variables observables qui sont une partie des variables motivant l'auto-sélection dans le processus d'adoption. De ce fait, seul le biais lié aux variables observables est supprimé [ROS 02, LEE 05].

Les producteurs anticipent leur rendement dans le cas de l'adoption des nouvelles technologies [IMB 94]. L'adoption de nouvelles technologies dépend des caractéristiques inobservées et des facteurs macroéconomiques affectant la productivité différentielle de la technologie comparée aux technologies traditionnelles [SUR 11]. Pour éliminer les biais liés aux caractéristiques observables ou inobservables et traiter le problème d'endogéneité de la variable traitement, plusieurs auteurs utilisent les variables instrumentales [IMB 94, ABA 01, ADE 05, DON 12]. Le rôle de la variable instrumentale est d'introduire une variation exogène dans la variable traitement en proposant une interprétation causale [ABA 01]. Cette méthode bien qu'elle prend en compte le problème de l'endogénéité liée à la sélection, n'est appliquée que dans le cas binaire.

\section{Modèle économétrique et analyse des données}

\subsection{Spécification de la méthode de frontière de production}

Les données sont issues de l'enquête du Projet d'Appui à la Politique Agricole (PAPA) pour le riz irrigué et de la Direction de l'Analyse, de la Prévision et des Statistiques Agricoles (DAPSA) pour le riz pluvial en 2017. L'échantillon est composé de 1200 ménages dont 526 qui choisissent le riz irrigué et 674 le riz pluvial.

L'estimation de la frontière de production est analysée à l'aide de la fonction transcendante logarithmique simplifiée. Cette méthode a, d'une part, l'avantage d'être flexible et n'impose aucune hypothèse restrictive sur la constante et l'élasticité de la fonction de production [DON 13]. D'autre part, elle permet de voir la combinaison entre deux facteurs de production sur le niveau de production. La fonction peut donc s'écrire comme suit :

$$
\ln Y_{i}=\beta_{0}+\sum_{j-1}^{5} \beta_{j} \ln X_{j}+\sum_{j-1}^{5} \sum_{l-1}^{5} \beta_{m} \ln X_{j} \ln X_{l}+\left(v_{i}-u_{i}\right) \quad \text { [1] }
$$

Avec : 
$Y_{i}$ : Production totale récoltée en kilogramme; $X_{1 i}$ : la superficie totale en ares cultivée ; $X_{2 i}$ : la quantité totale de semences utilisée en kilogrammes; $X_{3 i}$ : la quantité d'engrais totale (npk et urée) utilisée en kilogrammes ; $X_{4 i}$ : la quantité de main-d'œuvre exprimée en personne/jour; $X_{5 i}$ : la quantité d'équipements utilisée et $\beta$ le vecteur de paramètres à estimer.

Pour estimer l'impact des nouvelles technologies rizicoles sur l'efficacité technique, l'utilisation de la forme fonctionnelle translog semble la plus adaptée. Ce choix est justifié par la spécification la plus appropriée entre la fonction Cobb-Douglas et celle translog. Ainsi, le rejet de l'hypothèse nulle est induit par le fait que $\lambda=69,19$ est supérieur à la valeur critique.

Le modèle de fonction de production stochastique prend la forme suivante :

$$
\begin{aligned}
& \ln Y_{i}=\beta_{1} \operatorname{lnsup}_{i}+\beta_{2} \operatorname{lnsem}_{i}+\beta_{3} \operatorname{lneng}_{i}+\beta_{4} \operatorname{lnmainoeuv}_{i}+\beta_{6} \operatorname{lnéquip}_{i}+\beta_{7} \operatorname{lnsup}_{i}{ }^{2}+\beta_{8} \operatorname{lnsem}^{2} \\
& +\beta_{9} \text { lneng }_{i}{ }^{2}+\beta_{10} \text { lnmainoeuv }_{i}{ }^{2}+\beta_{11} \text { lnéquip }_{i}{ }^{2}+\beta_{12} \operatorname{lnsup}_{i} * \operatorname{lnsem}_{i}+\beta_{13} \operatorname{lnsup}_{i}
\end{aligned}
$$

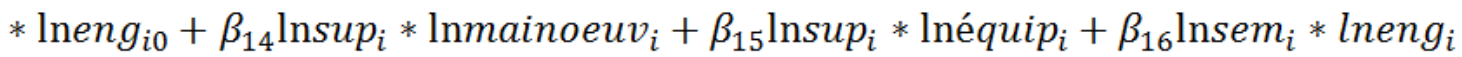

$$
\begin{aligned}
& +\beta_{17} \operatorname{lnsem}_{i} * \operatorname{lnmainoeuv}_{i}+\beta_{18} \operatorname{lnsem}_{i} * \operatorname{lnéquip}_{i}+\beta_{19} \operatorname{lneng}_{i} * \operatorname{lnmainoeuv}_{i} \\
& +\beta_{20} \operatorname{lneng}_{i} * \text { lnéquip }_{i}+\beta_{21} \operatorname{lnmainoeuv}{ }_{i} * \operatorname{lnéquip}_{i}+\epsilon_{i}
\end{aligned}
$$

$\ln Y_{i}$ est le log de la production rizicole en tonnes du ménage $i$. Les intrants (la superficie, les semences, engrais npk et urée, la main d'œuvre et les équipements agricoles) sont exprimés en log. Les paramètres $\beta_{1}$ à $\beta_{21}$ sont à estimer et $\epsilon_{i}$ désigne le terme d'erreur comprenant un terme de bruit $v_{i}$ et une inefficacité technique $u_{i}$.

Avec le modèle à choix multinomial, chaque individu $i$ fait un choix sur un ensemble de deux ou trois traitements. Soit $E V_{i j}$ correspond à l'utilité indirecte obtenue en sélectionnant un traitement $j$ allant de $j=0,1,2$ et 3 .

$$
E V_{i j}=z_{i}^{\prime} \alpha_{j}+\sum_{k=1}^{j} \delta_{j k} l_{i k}+\eta_{i j}
$$

Où $z_{i}$ correspond à des covariables exogènes pour lesquelles les paramètres associés $\alpha_{j}$ et $\eta_{i j}$ qui sont des termes d'erreur distribués de manière indépendante et identique, $E V_{i j}$ comprend un facteur latent $l_{i j}$ qui intègre des caractéristiques non observées communes au choix de traitement et au résultat de l'individu $i . l_{i j}$ est supposé être indépendant de $\eta_{i j}$. On considère que $j=1$ désigne le groupe de contrôle avec $E V_{i 0}=0$.

Soit $d_{j}$ les variables qui représentent le choix de traitement observé avec $d_{i}=\left(d_{i 1}, d_{i 2}, d_{i j}\right)$.

La probabilité de traitement peut s'écrire :

$$
p_{r}\left(d_{i} / z_{i}, l_{i}\right)=g\left(z_{i}^{\prime} \alpha_{1}+\sum_{k=1}^{j} \delta_{1 k} l_{i k}, z_{i}^{\prime} \alpha_{2}+\sum_{k=1}^{j} \delta_{2 k} l_{i k}, \ldots, z_{i}^{\prime} \alpha_{j}+\sum_{k=1}^{j} \delta_{j k} l_{i k}\right)
$$

Où $g$ est une distribution de probabilité multinomiale appropriée.

L'équation du résultat attendu pour l'individu $i, i=1,2, \ldots, N$, est formulée comme suit :

$$
\mathrm{E}\left(y_{i}\right)=\mu\left(z_{i}^{\prime} \beta+\sum_{j=1}^{j} \gamma_{j} d_{i j}+\sum_{j=1}^{j} \lambda_{j} l_{i j}\right)
$$

$\mathrm{E}\left(y_{i}\right)$ est une fonction de chacun des facteurs latents $l_{i j}$, c'est-à-dire que le résultat est affecté par des caractéristiques non observables qui affectent également la sélection dans le traitement. 


\subsection{Analyse des résultats}

L'analyse de la similitude des deux groupes montre qu'il y a une différence entre la moyenne des traités et celle des non-traités ${ }^{1}$. Au niveau de traitement T1, il y a 273 ménages qui sont dans le groupe traité et 927 ménages dans le groupe non-traité. Pour le niveau de traitement T2, les adoptants sont 357 ménages et les non-adoptants 843 ménages. Pour le niveau de traitement T3, le nombre d'adoptants est de 112 ménages et 1088 ménages non-adoptants.

Le tableau 1 ci-après présente les résultats du modèle probit de l'estimation des déterminants de l'adoption des nouvelles techniques rizicoles sur trois niveaux de traitements.

Nombre d'observations $=1124$

Wald ch2 (27) $=1787,59$

Prob $>$ chi $2=0.0000$

\begin{tabular}{|c|c|c|c|}
\hline Variables & Coef. & Std. En & $\mathrm{P}>|\mathrm{z}|$ \\
\hline & \multicolumn{3}{|c|}{ T1 (l'engrais) } \\
\hline Contrat & 1,075 & 0,782 & 0,169 \\
\hline Crédit & 1,914 & 0,815 & $0,019 * *$ \\
\hline M_OP & 1,026 & 0,291 & $0,000 * * *$ \\
\hline Assur & 0,788 & 0,668 & 0,238 \\
\hline Féminin & 0,333 & 0,314 & 0,289 \\
\hline Célibataire & 0,051 & 0,803 & 0,949 \\
\hline Age_cm & $-0,001$ & 0,007 & 0,907 \\
\hline Taill_ménag & 0,011 & 0,018 & 0,530 \\
\hline Accès_tech & 4,455 & 1,022 & $0,000 * * *$ \\
\hline \multirow[t]{2}{*}{ Cons } & $-1,036$ & 0,456 & $0,023 * *$ \\
\hline & \multicolumn{3}{|c|}{ T2 (engrais et semences améliorées) } \\
\hline Contrat & 0,440 & 0,816 & 0,589 \\
\hline Crédit & 3,912 & 0,781 & $0,000 * * *$ \\
\hline M_OP & 1,912 & 0,300 & $0,000 * * *$ \\
\hline Assur & 2,111 & 0,641 & $0,001 * * *$ \\
\hline Féminin & $-0,699$ & 0,430 & 0,104 \\
\hline Célibataire & $-1,455$ & 0,803 & 0,315 \\
\hline Age_cm & $-0,015$ & 0,009 & $0,092 *$ \\
\hline Taill_ménag & $-0,023$ & 0,021 & 0,312 \\
\hline Accès_tech & 6,803 & 1,018 & $0,000 * * *$ \\
\hline \multirow[t]{2}{*}{ Cons } & $-0,950$ & 0,532 & $0,074 *$ \\
\hline & \multicolumn{3}{|c|}{ T3 (engrais, semences améliorées et équipement motorisé) } \\
\hline Contrat & 1,859 & 0,790 & $0,016 * *$ \\
\hline Crédit & 3,479 & 0,809 & $0,000 * * *$ \\
\hline M_OP & 2,223 & 0,332 & $0,000 * * *$ \\
\hline Assur & 2,777 & 0,648 & $0,000 * * *$ \\
\hline Féminin & $-0,948$ & 0,603 & 0,116 \\
\hline Célibataire & $-0,358$ & 1,318 & 0,786 \\
\hline Age_cm & $-0,021$ & 0,11 & $0,056 *$ \\
\hline Taill_ménag & 0,016 & 0,26 & 0,547 \\
\hline Accès_tech & 4,412 & 1,043 & $0,000 * * *$ \\
\hline Cons & $-2,815$ & 0,180 & $0,000 * * *$ \\
\hline
\end{tabular}

Note : ***;** et $*$ significativité de $1 \% ; 5 \%$ et $10 \%$

Tableau 1. Variables déterminants de l'adoption des technologies selon le niveau de traitement Source : Auteurs, à partir des données de l'enquête du PAPA et de la DAPSA (2017)

Les résultats montrent que le modèle est globalement significatif au seuil de $1 \%$. L'adoption du niveau de traitement T1 (engrais) est déterminée par le crédit, l'appartenance à une organisation

\footnotetext{
${ }^{1}$ Voir Tableau 1 en Annexe
} 
paysanne (m_OP) et l'accès aux nouvelles technologies (accès_tech). Les déterminants du traitement T2 (engrais et semences améliorées) sont le crédit, l'appartenance à une organisation paysanne (m_OP), l'assurance, l'accès aux technologies et l'âge du chef de ménage (age_cm). L'adoption du traitement T3 (engrais, semences améliorées et équipement motorisé) est fortement influencée par les variables contrat, crédit, appartenance une organisation paysanne, assurance, accès aux nouvelles technologies et l'âge du chef de ménage.

Le coefficient de la variable crédit est positif et significatif pour le traitement $\mathrm{T} 1$ au seuil de $5 \%$ et pour les traitements $\mathrm{T} 2$ et $\mathrm{T} 3$ au seuil de 1\%. L'obtention d'un crédit par les riziculteurs joue un rôle favorable à l'adoption des trois niveaux de traitement. Ce résultat s'explique par le fait que la détention d'un crédit favorise l'adoption de nouvelles technologies. Ce résultat corrobore celui de Hailu et al. (2014), pour qui l'accès au crédit permet de renforcer l'utilisation de certains intrants.

Les variables appartenance à une organisation paysanne (m_OP), accès aux technologies (accès_tech) et assurance impactent positivement et significativement l'adoption des trois niveaux de traitement au seuil de $1 \%$. Ce résultat montre qu'au Sénégal, les organisations paysannes jouent un rôle important dans l'incitation à l'adoption des nouvelles technologies. Baumüller [BAU 12] soutient que la sensibilisation au sein des organisations paysannes est importante dans les décisions d'adopter une nouvelle technologie en agriculture. De plus, les incertitudes liées à l'adoption d'une nouvelle technologie font que la souscription à une assurance permette au producteur de se protèger des risques encourus. Selon Udry [UDR 10], les agriculteurs non protégés par une assurance ou disposant d'une assurance imparfaite seront plus réticents à un statut d'adoption.

En revanche, la variable âge du chef de ménage (age_cm) impacte négativement l'adoption des trois niveaux de traitements $\mathrm{T} 1, \mathrm{~T} 2$ et $\mathrm{T} 3$. Ce résultat révèle que les personnes âgées sont moins incitées à l'adoption de nouvelles technologies. Chirwa [CHI 05] avait montré que les agriculteurs relativement âgés sont les plus réticents à l'adoption des nouvelles technologies.

Nombre d'observations $=1124$

Wald ch2 (27) $=1787,59$

Prob $>$ chi $2=0.0000$

\begin{tabular}{|c|c|c|c|}
\hline Variables & Coef. & Std. Err. & $\mathrm{P}>|\mathrm{z}|$ \\
\hline T1 (engrais) & 0,015 & 0,016 & 0,370 \\
\hline T2 (engrais et semences améliorées) & 0,097 & 0,015 & $0,000 * * *$ \\
\hline T3 (engrais, sem_amélioré et équip_moto) & 0,121 & 0,022 & $0,000 * * *$ \\
\hline Nbre_parcelle & 0,004 & 0,002 & 0,153 \\
\hline Actv_principale & $-0,004$ & 0,019 & 0,807 \\
\hline Féminin & $-0,327$ & 0,017 & $0,000 * * *$ \\
\hline Célibataire & $-0,348$ & 0,044 & $0,000 * * *$ \\
\hline Marié_poly & $-0,372$ & 0,015 & $0,000 * * *$ \\
\hline Alphab & 0,273 & 0,203 & $0,000 * * *$ \\
\hline Auc_éduc_for & 0,217 & 0,016 & $0,000 * * *$ \\
\hline Age_cm & $-0,003$ & 0,000 & $0,000 * * *$ \\
\hline Saison_cult & $-0,521$ & 0,019 & $0,000 * * *$ \\
\hline Taill_ménag & $-0,003$ & 0,001 & $0,000 * * *$ \\
\hline Cons & $-3,634$ & 0,056 & $0,000 * * *$ \\
\hline /lnalpha & 3,903 & 0,046 & $0,000 * * *$ \\
\hline /lambda_T1 & 0,008 & 0,013 & 0,498 \\
\hline /lambda_T2 & $-0,011$ & 0,010 & 0,287 \\
\hline /lambda_T3 & $-0,014$ & 0,011 & 0,192 \\
\hline Alpha & 49,575 & 2,296 & \\
\hline
\end{tabular}

Note : ***;** et * significativité de $1 \% ; 5 \%$ et $10 \%$

Tableau 2 : Impact de l'adoption des nouvelles technologies sur l'efficacité technique

Source: Auteurs, à partir des données de l'enquête du PAPA et de la DAPSA (2017) 
Le tableau 2 ci-dessus présente l'impact de l'adoption des nouvelles technologies rizicoles sur l'efficacité technique. Le modèle est globalement significatif au seuil de $1 \%$ pour les trois niveaux de traitement. Le premier niveau de traitement T1 n'a pas d'impact sur l'efficacité technique du riziculteur.

L'adoption du niveau de traitement T2 impacte de 9,7\% l'efficacité technique des riziculteurs sénégalais au seuil de $1 \%$. Donc, la combinaison de l'engrais et des semences améliorées permet d'augmenter le niveau d'efficacité technique de 9,7\%. Ce résultat est similaire à celui de Hailu et al. [HAI 14], qui montrent que l'adoption des engrais chimiques et des semences à haut rendement augmentent le revenu des agriculteurs au Nord de l'Ethiopie.

L'adoption du traitement T3 permet d'augmenter le niveau d'efficacité technique de $12,1 \%$. Ce résultat montre que les riziculteurs qui utilisent la combinaison de l'engrais et des semences améliorées ont moins d'efficacité technique que ceux qui utilisent une combinaison d'engrais, de semences améliorées et d'équipement motorisé. Ainsi, la différence d'efficacité technique entre ceux qui utilisent le traitement $\mathrm{T} 2$ et le traitement $\mathrm{T} 3$ est de 2,4 points de pourcentage. Ces résultats sont identiques à ceux de Mendola [MEN 07] et Seck et al., [SEC 12] qui soutiennent que l'adoption des nouvelles technologies permette l'accroissement de la productivité. A la différence de ces résultats, notre approche méthodologique prend en compte une regression logistique multinomiale.

En outre au seuil de $1 \%$, la variable féminin impacte négativement l'efficacité technique de $32,7 \%$. Ce résultat montre que les rendements des exploitations rizicoles gérées par les femmes sont relativement moins efficaces que celles gérées par des hommes. Les variables célibataire et marié_polygamme (marié_polyg) impactent négativement l'efficacité technique respectivement de $34,8 \%$ et $37,2 \%$. La variable âge du chef de ménage (age_cm) réduit l'efficacité technique de $0,3 \%$. Ce résultat corrobore avec celui d'Alene et Manyong [ALE 06] qui montrent que le statut matrimonial impacte sur la manière d'adopter certaines nouvelles technologies. La taille du ménage (taille_menag) impacte négativement l'efficacité technique de $0,3 \%$. Ce résultat avance que l'efficacité dans la production des familles nombreuses est relativement faible car une bonne partie de la main d'œuvre n'est pas utilisée à l'exploitation rizicole. Le processus de mécanisation en termes d'équipements motorisés impacte donc sur le nombre de personnes dans le foyer.

Néanmoins, certaines variables telles que l'alphabétisation et aucune éducation formelle (auc_édu_formelle) impactent positivement l'efficacité technique respectivement de $27,3 \%$ et $21,7 \%$ au seuil de $1 \%$.

\section{Conclusion}

Le Sénégal est l'un des pays d'Afrique de l'Ouest où la consommation de riz est la plus importante. Sa production nationale couvre entre 20 à $30 \%$ la demande en riz du pays [MIN 14]. Ce déficit de production a fait l'objet de plusieurs interventions de l'Etat à travers des programmes sans pour autant permettre de réaliser l'objectif d'autosufisance. Cependant, la production du riz varie d'une année à l'autre sous l'influence de facteurs divers tels que les conditions pluviométriques, l'etat des sols, la disponibilité des intrants, la qualité des semences et l'intensité de la mécanisation. C'est dans ce cadre que l'amélioration des intrants à travers les opportunités offertes par les nouvelles technologies rizicoles devient approprier.

Cet article met en évidence les facteurs déterminants de l'adoption des nouvelles technologies (les engrais, les semences améliorées et les équipements motorisés). Parmi ces déterminants, les variables crédit, membre d'une organisation paysanne et accès aux technologies (affectent positivement le statut d'adoption des trois niveaux de traitements alors que la variable assurance influence seulement l'adoption des traitements T2 et T3. La variable âge du chef de ménage impacte négativement le statut d'adoption, car les personnes relativement âgées sont plus réticentes à l'adoption des innovations que les personnes moins âgées. 
Nos résultats révèlent aussi que l'efficacité technique augmente avec le niveau de traitement. En effet, lorsque le riziculteur adopte le traitement T1, l'impact sur l'efficacité technique n'est pas significatif. En revanche, lorsque celui-ci adopte le traitement T2 (engrais et semences), l'impact sur l'efficacité technique est de $9,7 \%$ au seuil de $1 \%$. Ce résultat montre que l'utilisation par le riziculteur d'une combinaison d'engrais et de semences améliorées impacte plus l'efficacité technique que l'utilisation unique de l'engrais (T1). Nous constatons aussi que l'adoption du traitement T3 (engrais, semences améliorées et équipement motorisé) impacte l'efficacité technique du riziculteur de 12,1\%, soit une hausse de 2,4 points de pourcentage par rapport au traitement T2. D'après ces résultats, les riziculteurs sénégalais qui utilisent de façon simultanée l'engrais, les semences améliorées et un équipement motorisé sont plus efficaces que ceux qui utilisent uniquement l'engrais et les semences améliorées.

En termes d'implications de politiques, cet article montre à quel point il est important d'investir sur les nouvelles technologies agricoles pour lutter contre la pauvreté et l'insécurité alimentaire. De ce fait, la mise en place de canaux efficaces de distribution de ces nouvelles technologies rizicoles et la formation des utilisateurs sont essentiels dans les stratégies d'autonomisation du monde rural. Cela permet d'adopter le niveau de traitement le plus élevé surtout lorsque d'autres innovations sont intégrées dans le processus de production agricole.

La principale limite de ce travail est qu'il existe plusieurs types de semences améliorées dont les caractéristiques ne sont pas identifiables à partir de la base de données. Par conséquent, l'une des perspectives de cette recherche est d'envisager d'exploiter des données relatives aux types de semences améliorées afin de différencier leur niveau d'efficacité. Au niveau de la méthode d'estimation, les méthodes d'évaluations d'impact comme la méthode instrumentale sont les plus appropriées, mais elles ne sont appliquées que dans le cas binaire alors que notre approche s'inscrit dans un cas multiple, d'où le recours au modèle de Deb et Trivedi [DEB 06].

\section{Bibliographie}

[ABA 01] ABADIE A., "Implementing matching estimators for average treatment effects in Stata", Stata Journal, 1(1), p.1-18, 2001.

[ADE 05] ADEKAMBI S.-A., «Impact de l'adoption des variétés améliorées de riz sur la scolarisation et la santé des enfants au Bénin: cas du département des Collines' » FSA, UAC, Bénin, 100p, 2005

[ADE 95] ADESINA A.-A., \& BAIDU-FORSON J., "Farmers' perceptions and adoption of new agricultural technology: evidence from analysis in Burkina Faso and Guinea", West Africa. Agricultural economics, 13(1), p. 1-9, 1995.

[ADE 93] ADESINA A.-A., ZINNAH M.-M., «Caractéristiques technologiques, perceptions des agriculteurs et décisions d'adoption: une application du modèle Tobit en Sierra Leone », Économie agricole , 9 (4), p. 297-311, 1993.

[ADE 05] ADEKAMBI S.|-A., Impact de l'adoption des variétés améliorées de riz sur la scolarisation et la santé des enfants au Bénin: cas du département des Collines', FSA, UAC, Bénin, 100p, 2005

[AIG 77] AIGNER D., LOVELL C.-K., SCHMIDT, P., "Formulation and estimation of stochastic frontier production function models", Journal of econometrics, 6(1), p. 21-37, 1977.

[ALE 06] ALENE A.-D., MANYONG V.-M., "Farmer-to-farmer technology diffusion and yield variation among adopters: The case of improved cowpea in northern Nigeria", Agricultural Economics 35: p. 203-211, 2006.

[AME 17] Ahmed, A., Paul Kawalek, J. et Kassem, M., "A Conceptual Model for Investigating BIM Adoption by Organisations", Lean and Computing in Construction Congress (LC3): Volume I Đ Proceedings of the Joint Conference on Computing in Construction (JC3), Heraklion, Greece, p. 447-455, 2017.

[BAN 06] BANDIERA O., et RASUL I., «Réseaux sociaux et adoption de la technologie dans le nord du Mozambique » Le journal économique, 116 (514), p.869-902, 2006. 
[BAN 08] BANQUE MONDIALE, World development report 2008: Agriculture for development, 2008.

[BAR 16] BARÁTH, L., \& FERTÖ, I., "Productivity and Convergence in European Agriculture", Journal of Agricultural Economics, 68(1), p. 228-248, 2017.

[BAT 95] BATTESE G.-E., COELLI T.-J., "A model for technical inefficiency effects in a stochastic frontier production function for panel data" Empirical economics, 20(2), p.325-332, 1995.

[BAU 12] BAUMÜLLER H., Facilitating agricultural technology adoption among the poor: The role of service delivery through mobile phones, Working paper Series 93, 2012.

[BOU 13] BOUTSEN S., et AERTSEN J., Peut-on nourrir l'Afrique de l'Ouest avec du riz, MO papers, (74), 2013.

[CHI 05] CHIRWA E.-W., "Adoption of fertiliser and hybrid seeds by smallholder maize farmers in Southern Malawi” Development Southern Africa, 22(1), p.1-12, 2005.

[CHU 20] CHURCHILL A., SEFA INEKWE J., IVANOVSKI K., "Convergence of R\&D intensity in OECD countries : Evidence since 1870”, Empirical Economics, 59(1), p. 295-306, 2020.

[CRO 03] CROPPENSTEDT A., DEMEKE M., MESCHI M.-M., "Technology adoption in the presence of constraints: the case of fertilizer demand in Ethiopia" Review of Development Economics, 7(1), p.58-70, 2003.

[DAK 16] DARKU, A. B., MALLA, S., \& TRAN, K. C., "Sources and Measurement of Agricultural Productivity and Efficiency in Canadian Provinces: Crops and Livestock : Sources and measurement of agricultural productivity and efficiency", Canadian Journal of Agricultural Economics, 64(1), p. 49-70, 2016.

[DEP 06] DEB P., TRIVEDI P.-K., "Specification and simulated likelihood estimation of a non-normal treatment-outcome model with selection: Application to health care utilization", The Econometrics Journal, 9(2), p.307$331,2006$.

[DEM 05] DEMEUSE M., MATOUL A., SCHILlINGS P., DENOOZ R., De quelle efficacité parle-t-on ?, Dans Vers une école juste et efficace, chapitre 1, p. 14-27, 2005.

[DON 13] DONKOH S.-A., AYAMBILA S., ABDULAI S., "Technical efficiency of rice production at the Tono irrigation scheme in northern Ghana", Experimental Agriculture, 2013.

[DON 12] DONTSOP NGUEZET P-M., OKORUWA V., ADENEGAN K-O., "Productivity Impact Differential of Improved Rice Technology Adoption Among Rice Farming Households in Nigeria", Journal of Crop Improvement, 26(1), p.1-21, 2012 .

[DUF 11] DUFLO E., KREMER M., ROBINSON J., "Nudging farmers to use fertilizer: Theory and experimental evidence from Kenya" The American Economic Review, 101:p. 2350-90, 2011

[DUV 17] DUVAL L., « Performance export des entreprises vitivinicoles françaises. Influence des stratégies et des politiques à l'exportation », Économie rurale, Vol.6 (n 362), p. 65-89, 2017.

[FA0 20] FAO, Programme mondial du recensement de l'agriculture 2020, VOLUME 2 Directives opérationnelles, 2020.

[FAR 57] FARREL J., "The measurement of Productive efficiency", Journal of the Royal Statistical Society, Series A, General 125. Part, (2), 252, 1957.

[FED 93] FEDER G., \& UMALI, D. L., "The adoption of agricultural innovations: a review" Technological forecasting and social change, 43(3-4), p. 215-239, 1993.

[GER 20] GERO A. A., «Innovation technologique, efficacité technique et convergence de la productivité agricole dans l’UEMOA », Ann. Univ. Lomé, série Sc. Eco. Et Gest., Volume XVI : p.55-67, 2020.

[GIL 90] GILLIS M., DWIGHT H.-P., ROEMER M., SNODGRASS D.-R., Economie du développement, De Boek, Nouveaux Horizons, 1990.

[HAI 14] HAILU B.-K., ABRHA B.-K., WELDEGIORGIS K.-A., "Adoption and impact of agricultural technologies on farm income: Evidence from Southern Tigray, Northern Ethiopia" International Journal of Food and Agricultural Economics (IJFAEC), 2(1128-2016-92058), p.91-106, 2014. 
[HEC 05] HECKMAN J.-J., VYTLACIL E., "Structural equations, treatment effects, and econometric policy evaluation, Econometrica, 73(3), p. 669-738, 2005.

[HEC 98] HECKMAN J-J., ICHIMURA H., TODD P., Matching As An Econometric, Evaluation Estimator, Review of Economic Studies (1998) 65, p. 261-294, 1998.

[HOC 18] HOCHSCHEID, É., \& HALIN, G., « L'adoption du BIM dans les agences d'architecture en France », In SHS Web of Conferences, Volume 47, 2018, SCAN'18 - 8e Séminaire de Conception Architecturale Numérique, EDP Sciences, 2018.

[IMB 94] IMBENS G. W., \& ANGRIST J. D., "Identification and estimation of Local Average" The Econometric Society, Vol. $62, \mathrm{n}^{\circ} 2$, p. 467-475, 1994

[KEA 09] KEANE J., PAGE S., KERGNA A., KENNAN J., Climate Change and Developing Country Agriculture: An Overview of Expected Impacts, Adaptation and Mitigation Challenges, and Funding Requirements, ICTSD-IPC Platform on Climate Change, Agriculture and Trade, International Centre for Trade and Sustainable Development, No.2, p. 1-49, 2009.

[KHA 17] KHAN F., SALIM R., BLOCH H., ISLAM N., "The public R\&D and productivity growth in Australia's broadacre agriculture : Is there a link ?", Australian Journal of Agricultural and Resource Economics, 61(2), p. 285-303, 2017.

[LAM 19] LAMPACH N., THE N.-T. NGUYEN T.-A., Technical Efficiency and the Adoption of Multiple Agricultural Technologies in the Mountainous Areas of Northern Vietnam, Available at SSRN: https://ssrn.com/abstract=3421434, 2019.

[LAM 16] LAMPACH N., MORAWETZ U.-B., "Credibility of propensity score matching estimates. An example from Fair Trade certification of coffee producers", Applied Economics, 48(44), p. 4227-4237, 2016.

[LEE 05] LEE J., "Effects of leadership and leader-member exchange on commitment", Leadership \& organization development journal, 2005.

[LEF 90] LEFEBVRE E., LEFEBVRE L.-A., COLIN D., «Facteurs d'adoption des nouvelles technologies de production dans les PME manufacturières », Revue internationale, P.M.E., 3 (2), p. 215-229, 1990.

[MAR 97] MARIS B., «Qu'est-ce que l'efficacité ?», Dans Bernard Charbonneau : une vie entière à dénoncer la grande imposture, p. 99-108, 1997.

[MAR 15] MARTEY E., DOGBE W., ETWIRE P.-M., WIREDU A.-N., "Impact of farmer mentorship project on farm efficiency and income in rural Ghana" Journal of Agricultural Science, 7(10), 79, 2015.

[MAY 10] MAYEN C.-D., BALAGTAS J.-V., ALEXANDER C.-E., "Technology adoption and technical efficiency: organic and conventional dairy farms in the United States", American Journal of Agricultural Economics, 92(1), p. 181$195,2010$.

[MEE 77] MEEUSEN, W. et VAN DEN BROECK, J., «Estimation de l'efficacité à partir des fonctions de production Cobb-Douglas avec erreur composée », Revue économique internationale, p. 435-444, 1977.

[MEN 07] MENDOLA M., «Adoption des technologies agricoles et réduction de la pauvreté: une analyse d'appariement du score de propension pour le Bangladesh rural », Politique alimentaire, 32 (3), p. 372-393, 2007.

[MIN 14] Ministère de l'Agriculture et de l'Equipement Rural, Document principal : Programme d'Accélération de la Cadence de l'Agriculture Sénégalaise (PRACAS), Volet agricole du Plan Sénégal Emergent (PSE), 2015.

[MUL 12] MULUGETA T., HUNDIE B., "Impacts of adoption of improved wheat technologies on households' food consumption in southeastern Ethiopia, Conference, August 18-24, 2012, Foz do Iguacu, Brazil 126766, International Association of Agricultural Economists, 2012.

[NGO 16] NGOM C.-A.-B., SARR F., FALL A.-A., « Mesure de l'efficacité technique des riziculteurs du bassin du fleuve Sénégal » Économie rurale, Agricultures, alimentations, territoires, (355), p. 91-105, 2016.

[NKA 04] NKAMLEU G.-B., "Productivity growth, technical progress and efficiency change in African agriculture", African Development Review, 16 (1), p. 203-222, 2004. 
[NUA 06] NUAMA E., "The Technical Efficiency of Rice Producers in Côte d'Ivoire: Extension Services in Question", Économie rurale, Volume 316, Issue 2, p. 36-47, 2010.

[NUA 06] NUAMA E., "Mesure de l'efficacité technique des agricultrices de cultures vivrières en Côted'Ivoire », Économie rurale. Agricultures, alimentations, territoires, (296), p. 39-53, 2006.

[OEC 17] OECD. Publishing, \& Food and Agriculture Organization, OECD-FAO agricultural outlook 2017-2026. OECD Publishing, 2017.

[PRA 14] Programme d'Accélération de la Cadence de l'Agriculture Sénégalaise PRACAS, Volet agricole du plan Sénégal emergent, 2014.

[ROG 03] ROGERS E.-M., Diffusion of innovation, 5 th ed., New York: Free Press, 2003.

[ROS 02] ROSENBAUM P.-R., Overt bias in observational studies. In Observational studies, Springer, New York, NY, p. 71-104, 2002.

[ROS 83] ROSENBAUM P.-R., Rubin D.-B., "The central role of the propensity score in observational studies for causal effects", Biometrika, 70(1), p. 41-55, 1983.

[ROU 15] ROUSSY C., RIDIER, A., CHAIB K., Adoption d'innovations par les agriculteurs: rôle des perceptions et des préférences. INRA, France, 2015.

[RUB 74] RUBIN D.-B., "Estimating causal effects of treatments in randomized and nonrandomized studies" Journal of Educational Psychology, 66(5), p. 688-701, 1974.

[SAV 98] SAVADOGO K., REARDON T., PIETOLA K., "Adoption of improved land use technologies to increase food security in Burkina Faso: relating animal traction, productivity, and non-farm income", Agricultural systems, 58(3), p. 441-464, 1998.

[SEC 12] SECK P.-A., DIAGNE A., MOHANTY S. WOPEREIS M.-C., « Des cultures qui nourrissent le monde 7: le riz », Sécurité alimentaire, 4 (1), p. 7-24, 2012.

[SUC 15] Succar, B. et Kassem, M. Macro-BIM adoption: Conceptual structures. Automation in Construction. Vol. 57, p. 64-79, 2015.

[SUR 11] SURI T., "Selection and comparative advantage in technology adoption" Econometrica, 79(1), p. 159-209, 2011.

[TOM 17] TOMA P., MIGLIETTA P. P., ZURLINI G., VALENTE D., PETROSILlO I., "A nonparametric bootstrap-data envelopment analysis approach for environmental policy planning and management of agricultural efficiency in EU countries", Ecological Indicators, Volume 83, p. 132-143, 2017.

[THI 95] THIRTL C., HADLEY D. TOWNSEND R.-F., "Policy-induced Innovation in Sub-Saharan African Agriculture: A Multilateral Malmquist Productivity Index Approach", Development Policy Review, 13 (4), p. 323-342, 1995.

[UDR 10] UDRY C., «L'économie de l'agriculture en Afrique: notes pour un programme de recherche », Journal africain d'économie agricole et des ressources, 5 (311-2016-5540), p. 284-299, 2010.

[VIL 15] VILLANO R., BRAVO-URETA B., SOLIS D., FLEMING E., Modern rice technologies and productivity in the Philippines: Disentangling technology from managerial gaps, Journal of Agricultural Economics, 66(1), p. 129-154, 2015.

[ZEL 98] ZELLER M., DIAGNE A., MATAYA C., "Market access by smallholder farmers in Malawi: Implications for technology adoption, agricultural productivity and crop income", Agricultural Economics, 19(1-2), p. 219-229, 1998. 


\begin{tabular}{|c|c|c|c|c|c|c|c|}
\hline \multirow[t]{2}{*}{ Variables } & \multirow[t]{2}{*}{ Définitions } & \multicolumn{2}{|c|}{ T1 (engrais) } & \multicolumn{2}{|c|}{$\begin{array}{ccc}\text { T2 } & \text { (engrais } & \text { et } \\
\text { semences améliorées) } & \end{array}$} & \multicolumn{2}{|c|}{$\begin{array}{l}\text { T3 (engrais, } \\
\text { semences améliorées et } \\
\text { équipements motorisés) }\end{array}$} \\
\hline & & Adop & Non-adop & adop & Non-adop & adop & Non-adop \\
\hline $\mathrm{Nbr} \_$parcelle & $\begin{array}{l}\text { Nombre de } \\
\text { parcelles } \\
\text { cultivé par } \\
\text { les } \\
\text { riziculteurs }\end{array}$ & $\begin{array}{l}2,02 \\
(272)\end{array}$ & $\begin{array}{l}2,16 \\
(924)\end{array}$ & $\begin{array}{l}1,67 * * \\
(356)\end{array}$ & $\begin{array}{l}2,33 * * \\
(840)\end{array}$ & $\begin{array}{l}3,20 * * * \\
(112)\end{array}$ & $\begin{array}{l}2,02 * * * \\
(1088))\end{array}$ \\
\hline contrat & $\begin{array}{l}\text { Bénéficié } \\
\text { d'un contrat } \\
\text { agricole } \\
1=\text { oui ; } \\
0=\text { non }\end{array}$ & $\begin{array}{l}0,03 \\
(927)\end{array}$ & $\begin{array}{l}0,04 \\
(273)\end{array}$ & $\begin{array}{l}0,05 \\
(357)\end{array}$ & $0,04(843)$ & $\begin{array}{l}0,18 * * * \\
(112)\end{array}$ & $\begin{array}{l}0,03 * * * \\
(1088)\end{array}$ \\
\hline crédit & $\begin{array}{l}\text { Bénéficié } \\
\text { d'un crédit } \\
\text { agricole } \\
1=\text { oui ; } \\
0=\text { non }\end{array}$ & $\begin{array}{r}0,04 * * * \\
(273)\end{array}$ & $\begin{array}{r}0,98 * * * \\
(927)\end{array}$ & $\begin{array}{l}0,16 * * * \\
(357)\end{array}$ & $\begin{array}{l}0,05 * * * \\
(843)\end{array}$ & $\begin{array}{l}0,18 * * * \\
(112)\end{array}$ & $\begin{array}{l}0,07 * * * \\
(1088)\end{array}$ \\
\hline M_OP & $\begin{array}{l}\text { 1=nombre } \\
\text { d'une } \\
\text { Organisation } \\
\text { paysanne ; } \\
0=\text { sinon }\end{array}$ & $\begin{array}{l}0,21 * * \\
(273)\end{array}$ & $\begin{array}{l}0,28 * * \\
(927)\end{array}$ & $\begin{array}{l}0,44 * * * \\
(357)\end{array}$ & $\begin{array}{l}0,19 * * * \\
(843)\end{array}$ & $\begin{array}{r}0,51 * * * \\
(112)\end{array}$ & $\begin{array}{l}0,24 * * * \\
(1088)\end{array}$ \\
\hline Act_prin & $\begin{array}{l}1=\text { agriculture } \\
; 0=\text { sinon }\end{array}$ & $\begin{array}{l}0,95 \\
(273)\end{array}$ & $\begin{array}{l}0,94 \\
(927)\end{array}$ & $\begin{array}{l}0,98 * * * \\
(357)\end{array}$ & $\begin{array}{l}0,93 * * * \\
(843)\end{array}$ & $\begin{array}{l}0,99 * * \\
(112)\end{array}$ & $\begin{array}{c}0,94 * * \\
(1088)\end{array}$ \\
\hline Féminin & $\begin{array}{l}1=\text { femme } \\
0=\text { sinon }\end{array}$ & $\begin{array}{l}0,12 * * \\
(273)\end{array}$ & $\begin{array}{l}0,07 * * \\
(927)\end{array}$ & $\begin{array}{l}0,06 * * \\
(357)\end{array}$ & $\begin{array}{l}0,10 * * \\
(843)\end{array}$ & $\begin{array}{l}0,04 * \\
(112)\end{array}$ & $\begin{array}{c}0,09 * \\
(1088) \\
\end{array}$ \\
\hline célibataire & $\begin{array}{l}1=\text { célibataire } \\
; 0=\text { sinon }\end{array}$ & $\begin{array}{c}0,14 \\
(273)\end{array}$ & $\begin{array}{c}0,01 \\
(927) \\
\end{array}$ & $\begin{array}{l}0,002 * * \\
(357)\end{array}$ & $\begin{array}{l}0,01 * * \\
(843)\end{array}$ & $\begin{array}{l}0,008 \\
(112)\end{array}$ & $\begin{array}{c}0,01 \\
(1088) \\
\end{array}$ \\
\hline Marié_polyg & $\begin{array}{l}\text { 1=marié } \\
\text { polygame } \\
0=\text { sinon }\end{array}$ & $\begin{array}{l}0,30 \\
(273)\end{array}$ & $\begin{array}{l}0,24 \\
(927)\end{array}$ & $\begin{array}{l}0,22 * * * \\
(357)\end{array}$ & $\begin{array}{l}0,31 * * * \\
(843)\end{array}$ & $\begin{array}{c}0,33 \\
(112)\end{array}$ & $\begin{array}{r}0,27 \\
(1088)\end{array}$ \\
\hline alphabétisation & $\begin{array}{l}1=\text { alphabétis } \\
\text { ation ; } \quad 0= \\
\text { sinon }\end{array}$ & $\begin{array}{l}0,07 \\
(273)\end{array}$ & $\begin{array}{l}0,08 \\
(927)\end{array}$ & $\begin{array}{l}0,13 \text { *** } \\
(357)\end{array}$ & $\begin{array}{l}0,06^{* * * *} \\
(843)\end{array}$ & $\begin{array}{l}0,24 * * * \\
(112)\end{array}$ & $\begin{array}{l}0,06^{* * * *} \\
(1088)\end{array}$ \\
\hline Age_cm & $\begin{array}{l}\text { Age du chef } \\
\text { de ménage }\end{array}$ & $\begin{array}{l}53,84 \\
(273) \\
\end{array}$ & $\begin{array}{l}52,75 \\
(927)\end{array}$ & $\begin{array}{l}51,66 * * \\
(357)\end{array}$ & $\begin{array}{l}53,57 * * \\
(843)\end{array}$ & $\begin{array}{l}51,84 \\
(112)\end{array}$ & $\begin{array}{l}53,12 \\
(1088)\end{array}$ \\
\hline Type_culture & $\begin{array}{l}1=\text { irrigation } ; \\
0=\text { sinon }\end{array}$ & $\begin{array}{c}0,42 \\
(273) \\
\end{array}$ & $\begin{array}{c}0,44 \\
(927) \\
\end{array}$ & $\begin{array}{l}0,74 * * * \\
(357)\end{array}$ & $\begin{array}{l}0,30 * * * \\
(843)\end{array}$ & $\begin{array}{l}0,98 * * * \\
(112)\end{array}$ & $\begin{array}{l}0,38 * * * \\
(1088)\end{array}$ \\
\hline Taille_ménage & $\begin{array}{l}\text { Le nombre } \\
\text { de personnes } \\
\text { que constitue } \\
\text { le ménage }\end{array}$ & $\begin{array}{l}9,59 \\
(273)\end{array}$ & $\begin{array}{l}9,23 \\
(927)\end{array}$ & $\begin{array}{l}8,59 * * * \\
(357)\end{array}$ & $\begin{array}{l}9,62 * * * \\
(843)\end{array}$ & $\begin{array}{l}10,41 * * \\
(112)\end{array}$ & $\begin{array}{l}9,20 * * \\
(1088)\end{array}$ \\
\hline
\end{tabular}

Note : niveau de significativité $1 \% ; 5 \%$ et $10 \%$ respectivement pour $* * * ; * *$ et *.

Tableau1. Caractéristiques socio-économiques des groupes d'adoptants selon le niveau de traitement Source : Auteurs à partir des données de l'enquête du PAPA et de la DAPSA (2017) 
Annexe 2 : Modèle empirique de l'efficacité technique

Le modèle de la frontière stochastique pour le producteur $\mathrm{i}$, avec $\mathrm{i} \in\{1,2,3,4, \ldots, \mathrm{n}\}$ s'écrit :

$$
\ln y_{i}=x_{i} \beta+v_{i}-u_{i}
$$

$y_{t}$, représente la production de l'exploitants i, $x_{i}$ désigne un vecteur d'intrants, c'est-à-dire le vecteur des coefficients associés à estimer, est le terme d'erreur qui capte les effets stochastiques qui ne sont pas sous le contrôle du riziculteur i et $u_{i}$ représente la variable aléatoire, positive ou nulle, traduisant l'inefficacité technique en termes de production de i. Ces deux dernières variables sont indépendantes et distribuées selon une loi normale tronquée à zéro avec une moyenne $\delta_{\mathrm{i}}$ et une variance $\sigma_{\mathrm{u}}{ }^{2}\left(\mathrm{~N}\left(\mathrm{ui}, \sigma_{\mathrm{u}}{ }^{2}\right)\right)$. La condition de non-négativité de i garantit que les observations ne peuvent pas se situer au-dessus de la frontière de production.

La production agricole du riziculteur $i$ peut être donnée par l'équation suivante :

$$
\ln \hat{y}_{i}=x_{i} \beta+v_{i}-u_{i}
$$

Cette équation représente la sortie de la frontière sans inefficacité :

$$
\ln \ddot{y}_{i}=x_{i} \beta+v_{i}
$$

Le ratio entre la production observée ( $\left.\ln \hat{y}_{i}\right)$ et celle estimée $\left(n \ddot{y}_{i}\right)$ sur la frontière de production du riziculteur parfaitement efficace utilisant le même vecteur d'intrants, $x_{i}$ donne l'estimation de l'efficacité technique. Ainsi, le niveau d'efficacité d'un riziculteur est obtenu de la façon suivante :

$$
\ln \mathrm{ET}_{i}=\frac{\ln \hat{\mathrm{y}}_{i}}{\ln \ddot{y}_{i}}=-u_{i}
$$

Le niveau d'efficacité a une valeur comprise entre zéro et un. L'objectif est de mesurer la production maximale du riziculteur en proportion de la production correspondant à celle de la frontière de production optimale avec les mêmes intrants. Les paramètres du modèle de la frontière sont estimés dans l'équation (1) par l'estimation du maximum de vraisemblance. La spécification de la fonction de production Trans-logarithmique (translog) est utilisée pour estimer la fonction de vraisemblance. 